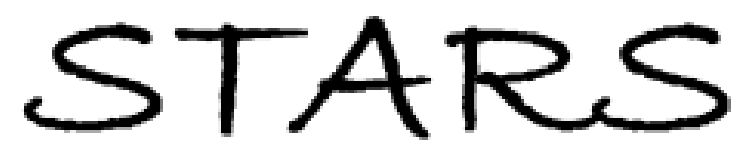

University of Central Florida

STARS

$1-1-2002$

\title{
Optimal rubbing angle for reflective in-plane-switching liquid crystal displays
}

Yubao Sun

Zhidong Zhang

Hongmei Ma

Xinyu Zhu

University of Central Florida

Shin-Tson Wu

University of Central Florida

Find similar works at: https://stars.library.ucf.edu/facultybib2000

University of Central Florida Libraries http://library.ucf.edu

This Article is brought to you for free and open access by the Faculty Bibliography at STARS. It has been accepted for inclusion in Faculty Bibliography 2000 s by an authorized administrator of STARS. For more information, please contact STARS@ucf.edu.

\section{Recommended Citation}

Sun, Yubao; Zhang, Zhidong; Ma, Hongmei; Zhu, Xinyu; and Wu, Shin-Tson, "Optimal rubbing angle for reflective in-plane-switching liquid crystal displays" (2002). Faculty Bibliography 2000s. 3496.

https://stars.library.ucf.edu/facultybib2000/3496

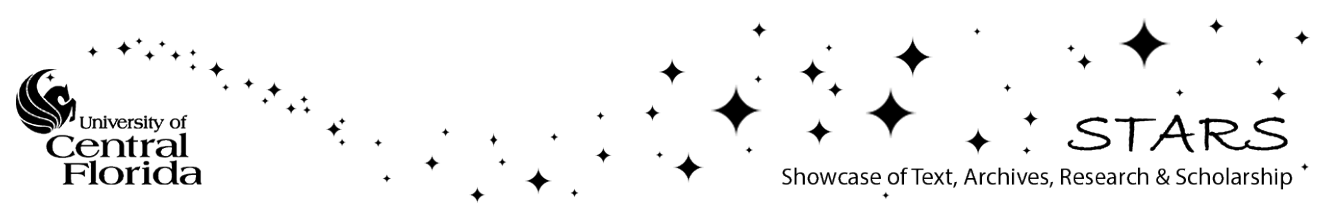




\section{Optimal rubbing angle for reflective in- plane-switching liquid crystal displays}

Cite as: Appl. Phys. Lett. 81, 4907 (2002); https://doi.org/10.1063/1.1532534

Submitted: 23 September 2002 . Accepted: 31 October 2002 . Published Online: 17 December 2002

Yubao Sun, Zhidong Zhang, Hongmei Ma, Xinyu Zhu, and Shin-Tson Wu

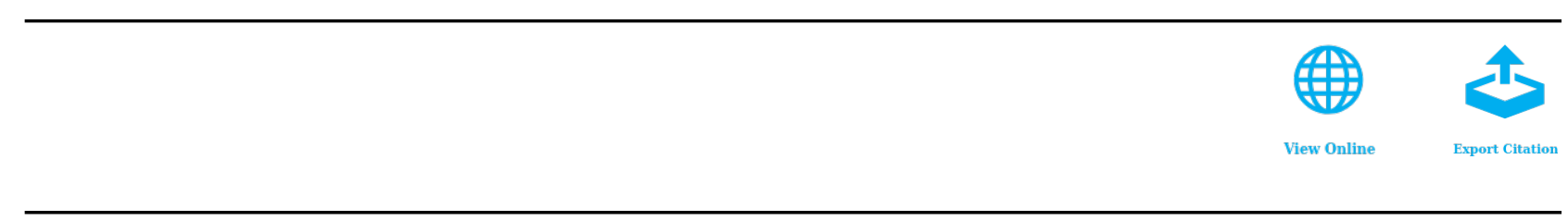

\section{ARTICLES YOU MAY BE INTERESTED IN}

Electro-optical characteristics and switching behavior of the in-plane switching mode Applied Physics Letters 67, 3895 (1995); https://doi.org/10.1063/1.115309

Anchoring energy and cell gap effects on liquid crystal response time Journal of Applied Physics 101, 103110 (2007); https://doi.org/10.1063/1.2734870

VOLTAGE-DEPENDENT OPTICAL ACTIVITY OF A TWISTED NEMATIC LIQUID CRYSTAL Applied Physics Letters 18, 127 (1971); https://doi.org/10.1063/1.1653593

Applied Physics Letters

Mid-IR and THz frequency combs special collection 


\title{
Optimal rubbing angle for reflective in-plane-switching liquid crystal displays
}

\author{
Yubao Sun, Zhidong Zhang, and Hongmei Ma \\ Department of Applied Physics, Hebei University of Technology, Tianjin, 300130, \\ People's Republic of China
}

Xinyu Zhu and Shin-Tson Wua)

School of Optics/CREOL, University of Central Florida, Orlando, Florida 32816

(Received 23 September 2002; accepted 31 October 2002)

\begin{abstract}
Rubbing angle effects on the response time and optical threshold voltage of the reflective in-plane-switching homogeneous liquid crystal displays were analyzed. As the rubbing angle increases, the rise time decreases except that its operating voltage also increases. The optimal rubbing angle is around $30^{\circ}-40^{\circ}$. (C) 2002 American Institute of Physics.
\end{abstract}

[DOI: $10.1063 / 1.1532534]$

Reflective liquid crystal displays (LCDs) ${ }^{1}$ offer several advantages over the transmissive ones in low power consumption, sunlight readability, and film-like image quality. The in-plane switching (IPS) mode has been used for largescreen transmissive LCDs because of its wide viewing angle. ${ }^{2-4}$ Recently, the IPS mode has been extended to reflective liquid crystal displays. ${ }^{5-8}$ As expected, wide viewing angle and high contrast ratio are obtained for the reflective IPS mode. However, the response time of the IPS mode is relatively slow as compared to other modes. ${ }^{9}$ In the IPS mode, the LC response time depends on the cell gap, rotational viscosity, twist elastic constant, applied electric field, and the rubbing angle $(\Phi) .{ }^{10}$ Among these parameters, the rubbing angle effect has not been analyzed in detail due to the difficulty in defining the optical transition threshold.

In this letter, we derived the IPS LC director's dynamic response by solving the Erickson-Leslie equation. This derivation is also valid to the transmissive IPS mode, although our emphasis is for reflective displays. The rubbing angle effects are described quantitatively.

Figure 1 shows the electrode configuration of the IPS mode under study. The comb-shaped electrodes are in the top substrate where $z=0$ in order to separate from the bottom reflector. The electrode gap is $\ell(\sim 10 \mu \mathrm{m})$ and width $\omega(\sim 5$ $\mu \mathrm{m})$. When backflow and inertial effects are ignored, the dynamics of liquid crystal director rotation is described by the following Erickson-Leslie equation: ${ }^{4,9}$

$$
\gamma_{1} \frac{\partial \phi}{\partial t}=K_{2} \frac{\partial^{2} \phi}{\partial z^{2}}+\varepsilon_{0}|\Delta \varepsilon| E^{2} \sin \phi \cos \phi .
$$

In Eq. (1), $\gamma_{1}$ is the rotational viscosity, $K_{2}$ is the twist elastic constant, $\Delta \varepsilon$ is the dielectric anisotropy, $E$ is the electric field strength, and $\phi$ is the LC rotation angle. The homogeneous LC layers having cell gap $d$ are along the $z$ axis.

In our analysis, we assume the surface anchoring strength is strong so that the bottom and top boundary layers are fixed at $\phi(0)=\phi(d)=\Phi$, where $\Phi$ is the LC alignment (or rubbing) angle with respect to the electrodes, as shown in Fig. 1. The middle layer has the maximum twist deformation

a)Electronic mail: swu@mail.ucf.edu angle $\phi(d / 2)=\phi_{m}$. Using these initial conditions, in a steady state the electric field-induced twist angle $\phi_{0}$ along the $z$ axis could be approximated as

$$
\phi_{0}(z)=\Phi+\left(\phi_{m}-\Phi\right) \sin \left(\frac{\pi z}{d}\right) .
$$

First, let us consider the relaxation process. We assume the electric field is removed instantaneously at time $t=0$. The relaxation time $\tau$ for returning from the activated state to the initial state has the following exponential form:

$$
\phi(z, t)=\Phi+\left(\phi_{m}-\Phi\right) \sin \left(\frac{\pi z}{d}\right) \exp (-t / \tau) .
$$

Substituting Eq. (3) into Eq. (1), we derive the free relaxation time

$$
\begin{aligned}
& -\frac{\gamma_{1}}{\tau}\left(\phi_{m}-\Phi\right) \sin (\pi z / d) \exp (-t / \tau) \\
& \quad=-\frac{K_{2} \pi^{2}}{d^{2}}\left(\phi_{m}-\Phi\right) \sin (\pi z / d) \exp (-t / \tau) .
\end{aligned}
$$

From Eq. (4), the relaxation time can be solved easily

$$
\tau_{\text {off }}=\frac{\gamma_{1} d^{2}}{\pi^{2} K_{2}} .
$$

From Eq. (5), the relaxation time is governed by the cell gap $(d)$ and the LC viscoelastic coefficient $\left(\gamma_{1} / \mathrm{K}_{2}\right)$, and is independent of the rubbing angle.

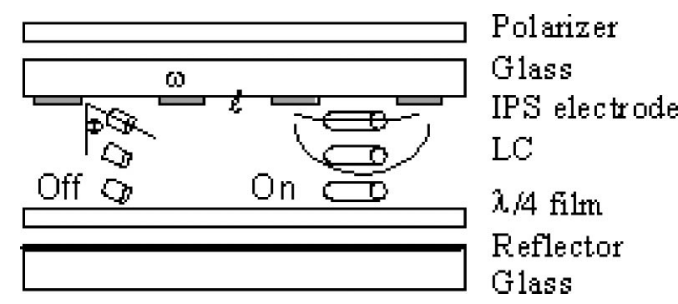

FIG. 1. Device structure of the reflective IPS LCD. The rubbing direction is at an angle $\Phi$ with respect to the IPS electrodes. $\ell=$ electrode gap and $\omega$ $=$ electrode width. 


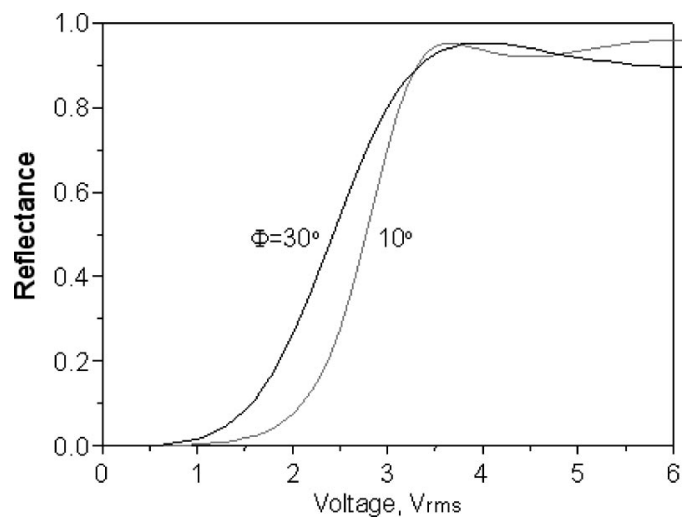

FIG. 2. Voltage-dependent reflectance of a reflective IPS homogeneous LC cell at $10^{\circ}$ and $30^{\circ}$ rubbing angles. $d=3 \mu \mathrm{m}, \Delta n=0.06 . \ell=10 \mu \mathrm{m}$, and $\lambda=550 \mathrm{~nm}$.

Next, we analyzed the switching-on process. The LC directors are described as ${ }^{11}$

$$
\phi(z, t)=\Phi+\left(\phi_{m}-\Phi\right) \sin \left(\frac{\pi z}{d}\right) \exp [(t-\tau) / \tau] .
$$

Substituting Eq. (6) in to Eq. (1), we find

$$
\begin{aligned}
\frac{\gamma_{1}}{\tau}\left(\phi_{m}-\Phi\right) \sin \left(\frac{\pi z}{d}\right) \exp [(t-\tau) / \tau] \\
=-K_{2} \frac{\pi^{2}}{d^{2}}\left(\phi_{m}-\Phi\right) \sin \left(\frac{\pi z}{d}\right) \exp [(t-\tau) / \tau] \\
\quad+\varepsilon_{0}|\Delta \varepsilon| E^{2} \sin (\Phi+x) \cos (\Phi+x) .
\end{aligned}
$$

In Eq. (7), the parameter $x$ is defined as

$$
x=\left(\phi_{m}-\Phi\right) \sin \left(\frac{\pi z}{d}\right) \exp [(t-\tau) / \tau] .
$$

Let $\bar{x}=\int_{0}^{d / 2} x d z$. At $\mathrm{t}=\tau, \bar{x}=2 / \pi\left(\phi_{m}-\Phi\right)$ where $\left(\phi_{m}-\Phi\right)$ represents the twisted angle of the middle LC layer under the exerted electric filed. Using Eq. (8), Eq. (7) can be rewritten as follows:

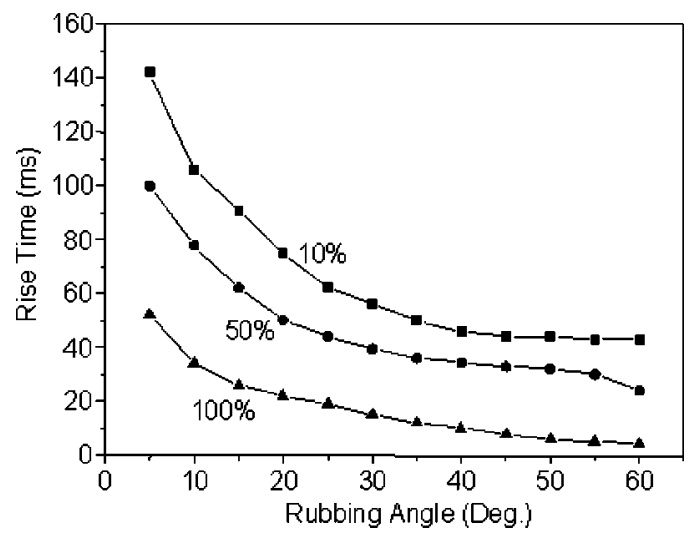

FIG. 3. Rubbing angle dependent rise time of a reflective IPS LCD. LC cell has homogeneous alignment and cell gap $d=3 \mu \mathrm{m}, \gamma_{1}=0.1 \mathrm{Pas}$ and $K_{2}$ $=7 \mathrm{pN}$.

$$
\begin{aligned}
\frac{\gamma_{1}}{\tau} \bar{x}= & -K_{2} \frac{\pi^{2}}{d^{2}} \bar{x}+\frac{1}{2} \varepsilon_{0}|\Delta \varepsilon| E^{2}[\sin (2 \Phi) \cos (2 \bar{x}) \\
& +\cos (2 \Phi) \sin (2 \bar{x})] .
\end{aligned}
$$

When the rubbing angle $\Phi=0$, the LC directors are perpendicular to the electric field and the Freedericksz transition exists. Under this circumstance, the turn-on time is

$$
\tau_{\mathrm{on}}=\frac{\gamma_{1}}{\varepsilon_{0}|\Delta \varepsilon| E^{2} \frac{\sin (2 \bar{x})}{2 \bar{x}}-\frac{\pi^{2}}{d^{2}} K_{2}} .
$$

In principle, Eq. (10) is not limited to the small signal regime. In the usual small angle approximation (i.e., the electric field is only slightly above threshold), $\bar{x} \ll 1$ and the rise time is reduced to the following commonly known equation:

$$
\tau_{\text {on }}=\frac{\gamma_{1}}{\varepsilon_{0}|\Delta \varepsilon| E^{2}-\frac{\pi^{2}}{d^{2}} K_{2}} .
$$

When the rubbing angle $\Phi \neq 0$, we can also obtain $\tau_{\text {on }}$ as

$$
\tau_{\text {on }}=\frac{\gamma_{1}}{\varepsilon_{0}|\Delta \varepsilon| E^{2}\left[\cos (2 \Phi) \frac{\sin (2 \bar{x})}{2 \bar{x}}+\sin (2 \Phi) \frac{\cos (2 \bar{x})}{2 \bar{x}}\right]-\frac{\pi^{2}}{d^{2}} K_{2}} .
$$

For a given electric field, $\bar{x}$ can be obtained from $\phi_{m}$ which, in turn, is calculated from the following elliptical equation: ${ }^{12}$

$$
\frac{E d}{2} \sqrt{\frac{\varepsilon_{0}|\Delta \varepsilon|}{K_{2}}} \sin \phi_{m}=\int_{\Phi}^{\phi_{m}} \frac{1}{\sqrt{1-\left(\sin \phi / \sin \phi_{m}\right)^{2}}} d \phi
$$

Strictly speaking, when the rubbing angle $\Phi \neq 0$ there exists no Freedericksz transition threshold. However, in a normally black IPS mode, the reflectance is proportional to the phase retardation $\delta=2(2 \pi d \Delta n / \lambda)$ of the LC cell as $R$ $\sim \sin ^{2}(\delta / 2)$. In the small voltage regime, the phase retarda- tion is small and $R$ exhibits a threshold-like transition. In our simulations, the rise time is defined based on the reflectance change from $R_{0}$ to $R_{90}$.

This optical threshold voltage $\left(V_{\mathrm{op}}\right)$ can be derived by assuming that the rise time is approaching infinity at $V$ $=V_{\mathrm{op}}$. Thus, the denominator in Eq. (12) should vanish

$$
\begin{aligned}
\varepsilon_{0}|\Delta \varepsilon| E^{2}\left[\cos (2 \Phi) \frac{\sin (2 \bar{x})}{2 \bar{x}}+\sin (2 \Phi) \frac{\cos (2 \bar{x})}{2 \bar{x}}\right] \\
-\frac{\pi^{2}}{d^{2}} K_{2} \rightarrow 0 .
\end{aligned}
$$

From Eq. (14), the optical threshold voltage is expressed as 


$$
V_{\mathrm{op}}=E \cdot \ell=\frac{\pi \ell}{d} \sqrt{\frac{K_{2}}{\varepsilon_{0}|\Delta \varepsilon|\left[\cos (2 \Phi) \frac{\sin (2 \bar{x})}{2 \bar{x}}+\sin (2 \Phi) \frac{\cos (2 \bar{x})}{2 \bar{x}}\right]}}
$$

From Eq. (15), when $\Phi=0$ and $\bar{x} \rightarrow 0$, the optical threshold is reduced to the Freedericksz threshold. ${ }^{13}$ As the rubbing angle is increased, the optical threshold voltage is gradually decreased. At $\Phi=45^{\circ}$, the optical threshold voltage has a minimum.

In our calculations for a reflective IPS mode, we have placed the interdigitated indium tin oxide electrodes in the top substrate and an aluminum reflector in the bottom substrate. A quarter-wave film is needed between the bottom LC substrate and the reflector in order to obtain the normally black mode. The TFT aperture ratio was not taken into account. The following LC cell parameters are used for calculations: $d=3 \mu \mathrm{m}, \gamma_{1}=0.1 \mathrm{Pas}, K_{2}=7 \mathrm{pN}, \Delta \varepsilon=7.8, \Delta n$ $=0.06, \ell=10 \mu \mathrm{m}$, and $\omega=5 \mu \mathrm{m}$. Figure 2 plots the voltage-dependent reflectance of the homogeneous LC cell at $\lambda=550 \mathrm{~nm}$ for two rubbing angles $\Phi=10^{\circ}$ and $30^{\circ}$. At $\Phi$ $=10^{\circ}$, the optical threshold $(R=10 \%)$ is $\sim 2.1 V_{\text {rms }}$ while at $\Phi=30^{\circ}$ the threshold is reduced to $\sim 1.6 V_{\text {rms }}$. The on-state voltage for $\Phi=30^{\circ}$ is slightly higher than that for $\Phi=10^{\circ}$.

The major advantage for the increased rubbing angle is on the improved rise time. Figure 3 shows the rubbing angle dependent rise time at three gray levels: $R=10 \%, 50 \%$, and $100 \%$; here $R$ stands for the normalized reflectance. As the rubbing angle increases, the rise time decreases. For example, the conventional IPS cell uses $\Phi \sim 10^{\circ}$. Its rise time for the $R=100 \%$ curve is $35 \mathrm{~ms}$. By merely changing the rubbing angle to $\Phi=30^{\circ}$, the rise time is reduced to $15 \mathrm{~ms}$.

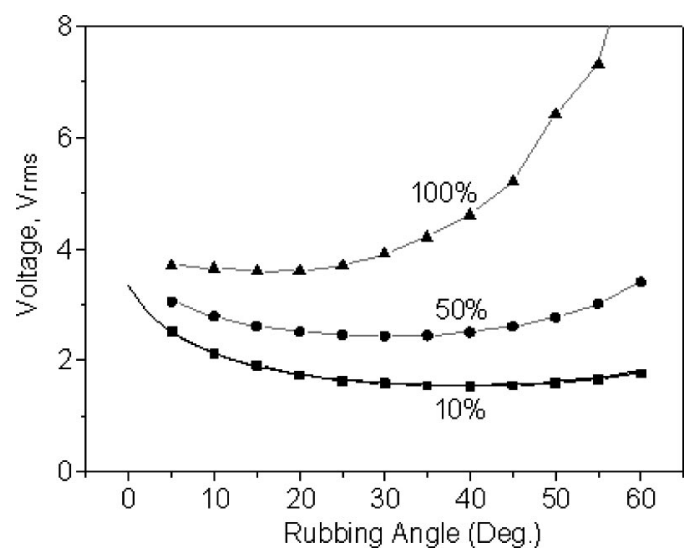

FIG. 4. Rubbing angle dependent optical threshold $(R=10 \%)$, gray level $(R=50 \%)$ and on-state $(R=100 \%)$ voltage. The fitting lines in $R=10 \%$ curve are calculated using Eq. (15).
As discussed in Eq. (5), the rubbing angle makes no effect to the decay time.

Although rubbing angle makes a significant effect on the rise time, we have to evaluate its impact on the operating voltage. Figure 4 plots the simulated results on the rubbing angle dependent operating voltage at three gray levels, $R$ $=10 \%, 50 \%$, and $100 \%$. The optical threshold voltage which corresponds to $R_{10}$ decreases as the rubbing angle $\Phi$ increases, reaches a minimum at $\Phi=45^{\circ}$ and then slowly bounces back. The analytical results using Eq. (15) overlap well with the square data points that are calculated without any approximation. From Fig. 4, the on-state voltage (corresponding to $R=100 \%$ ) increases noticeably as $\Phi>35^{\circ}$. If the maximum voltage of the amorphous silicon thin film transistor is $5 V_{\text {rms }}$, then the optimal rubbing angle should be set in the $30^{\circ}-40^{\circ}$ range for achieving faster rise time.

In conclusion, we have analyzed the rubbing angle effects on the rise time and operating voltages of a reflective in-plane-switching homogeneous cell. In general, the rise time decreases as the rubbing angle increases. The tradeoff is its increased on-state voltage. The optimal rubbing angle occurs at around $30^{\circ}-40^{\circ}$. The analyses developed here can also apply to the transmissive IPS LC cells.

The UCF group is supported by AFOSR under Contract No. 49620-01-1-0377.

${ }^{1}$ S. T. Wu and D. K. Yang, Reflective Liquid Crystal Displays (Wiley, Chichester, 2001).

${ }^{2}$ R. Kiefer, B. Weber, F. Windsceid, and G. Baur, Proceedings of the 12th International Display Research Conference, Hiroshima, Japan, 1992, p. 547.

${ }^{3}$ M. Oh-e and K. Kondo, Appl. Phys. Lett. 67, 3895 (1995).

${ }^{4}$ M. Oh-e, M. Yoneya, and K. Kondo, J. Appl. Phys. 82, 528 (1997).

${ }^{5}$ S. H. Lee, S. H. Hong, H. Y. Kim, D. S. Seo, G. D. Lee, and T. H. Yoon, Jpn. J. Appl. Phys., Part 1 40, 5334 (2001).

${ }^{6}$ S. Oka, M. Kimura, and T. Akahane, Appl. Phys. Lett. 80, 1847 (2002).

${ }^{7}$ K. H. Fan Chiang, S. T. Wu, and S. H. Chen, Jpn. J. Appl. Phys., Part 141 , 4577 (2002)

${ }^{8}$ Y. Sun, H. Ma, Z. Zhang, and S. T. Wu, J. Appl. Phys. 92, 1956 (2002).

${ }^{9}$ M. Oh-e and K. Kondo, Liq. Cryst. 22, 379 (1997).

${ }^{10}$ F. Di Pasquale, H. Deng, F. A. Fermandez, S. E. Day, J. B. Davies, M. T. Johnson, A. A. van der Put, J. M. A. van de Eerenbeemd, J. A. M. M. van Haaren, and J. A. Chapman, IEEE Trans. Electron Devices 46, 661 (1999).

${ }^{11}$ P. Pieranski, F. Brochard, and E. Guyon, J. Phys. (Paris) 34, 35 (1973).

${ }^{12}$ S. H. Lee, J. G. You, H. Y. Kim, D. S. Lee, S. K. Kwon, H. S. Park, and C. K. Kim, SID Tech. Digest 28, 711 (1997).

${ }^{13}$ C. J. Yu, J. H. Kim, and S. D. Lee, Jpn. J. Appl. Phys., Part 1 41, 5298 (2002). 